Case Series

\title{
Case series: gastric emphysema and emphysematous gastritis with air in portal venous system
}

\section{Introduction}

Presence of intramural gas in the stomach, gastric emphysema (GE) and emphysematous gastritis (EG) are rare but alarming radiological finding. The clinical course of GE and EG depends on underlying etiologies such as emphysematous gastritis (infected by gas forming organism), gastric ischemia, increased intraluminal pressure, chemical or mechanical injuries, or perforated gastric ulcer. We report the case of gastric emphysema in a 92year old elderly female who presented with gastric ischemia triggered by sliding ventral hernia and the case of emphysematous gastritis in a 74year elderly nursing home resident with acute respiratory failure, with bacteremia and septic shock most likely from right thigh abscess.

\section{Case I}

A 92-year-old African American lady with history of small bowel obstruction, status post diversion colostomy presented with one day duration of vague abdominal pain and multiple episodes of emesis after having heavy meal. Physical examination is remarkable for large reducible ventral hernia, without sign of peritonitis or sepsis. On the initial presentation mild leukocytosis and lactic acidosis were noted. A computed tomography (CT) scan of the abdomen showed gastric pneumatosis along with large left ventral hernia containing a portion of stomach and small bowel (Figure 1). No intra-abdominal free air was reported. Patient was treated conservatively with bowel rest, intravenous fluid, empiric antibiotics and anti-emetic medication. The next day lactic acid was trended down to normal. Endoscopy showed severely nodular erythematous mucosa with whitish exudate seen in body of stomach. The stomach was also appeared to be twisted along the axis where ventral hernia was found (Figure 2). In considering of the patient's age and multiple comorbidities, conservative management was appreciated. She was discharged home without complication.

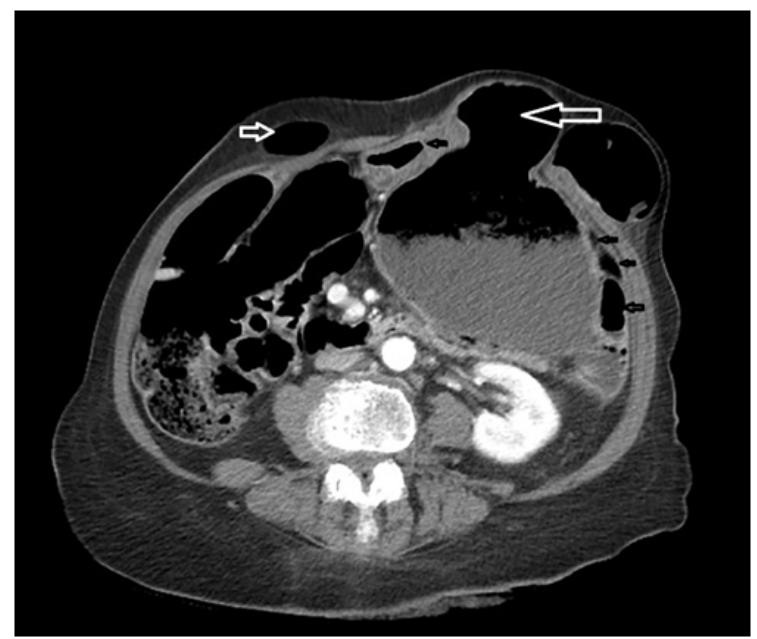

Figure I CT scan of the abdomen (a) small dark arrow showing interval appearance of gastric mural pneumatosis, (b) big white arrow showing ventral sliding hernia, (c) small white arrow showing status post subtotal colectomy and right anterior colostomy, and (d) fluid-containing distal thoracic esophagus, dilated stomach containing fluid and particulate matter.
Volume 9 Issue I - 2018

Lin Kyawzaw,' Ofori Emmanuel, ${ }^{2}$ Linn Sandar, ${ }^{2}$ Ofosu Andrew, ${ }^{2}$ Lin Aung Naing,' Fuksbrumer Moshe, ${ }^{3}$ Reddy Madhavi ${ }^{2}$

'Department of Internal Medicine, The Brooklyn Hospital Center, USA

${ }^{2}$ Gastroenterologist Fellow, The Brooklyn Hospital Center, USA

${ }^{3}$ Department of Radiology, The Brooklyn Hospital Center, USA

Correspondence: Kyawzaw Lin, Department of Medicine, The Brooklyn Hospital Center, 1733 West Ist Street, Brooklyn, NY I I223, USA, Tel 315-664-1916,

Email dr.kyawzawlin2015@gmail.com

Received: January II, 2018 | Published: February 08, 2018

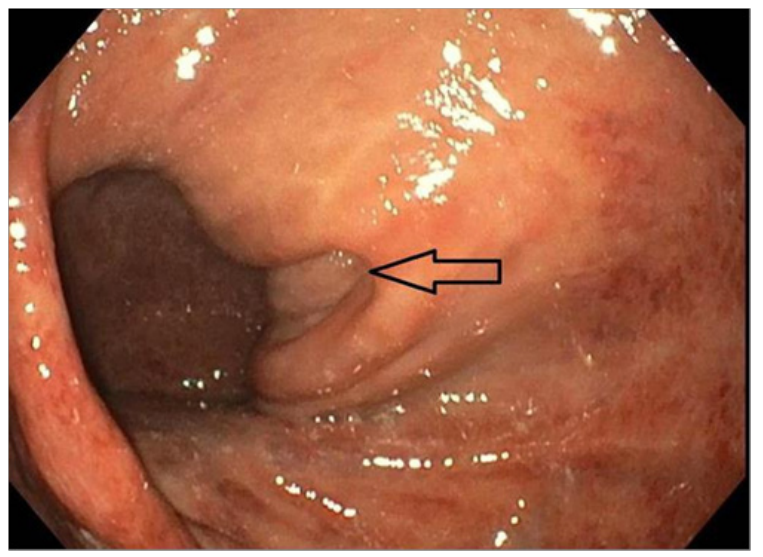

Figure 2 EGD showed severely nodular erythematous mucosa with whitish exudate seen in body of stomach. The stomach was also appeared to be twisted along the axis where ventral hernia was found.

\section{Case 2}

A 74-year- old nursing home resident male with CAD status post cardiac pacemaker, diabetic nephropathy current on hemodialysis and CVA without residual side-effects presented with fever of $102 \mathrm{~F}$ and altered mental status. Later he was admitted to the intensive care unit for acute respiratory failure, with bacteremia and septic shock most likely from right thigh abscess. On arrival, he was hypotensive and un-responsive to intravenous fluids. After rapid intubation and central line placement, he was started on aggressive hydration, intravenous vancomycin, pipercillin with tazobactam and pressors. White count 36.6 with left shift, metabolic acidosis on arterial gas with $\mathrm{pH}$ of 7.13 and lactic acid trending at 2.5 to 4.5 were noted. Later, a CT scan of 
right hip showed acute osteomyelitis involving the posterior aspect of the proximal to mid right femur with overlying abscess cavity. A CT scan (abdomen and pelvis) showed presence of gastric wall pneumatosis, transverse duodenal diverticulosis, $2.6 \mathrm{~cm}$ small bowel lipoma and air/gas lucencies noted within the distal main and left portal veins (Figures $3 \& 4$ ). EGD showed mild gastritis in antrum of stomach and single smooth nodule measuring $5-6 \mathrm{~mm}$ in size in the antrum (Figure 5). Owing to multiple co-morbidities, septic shock and unstable condition, conservative optimal management was made after the extensive family meeting. Later, the family opted for palliative care.

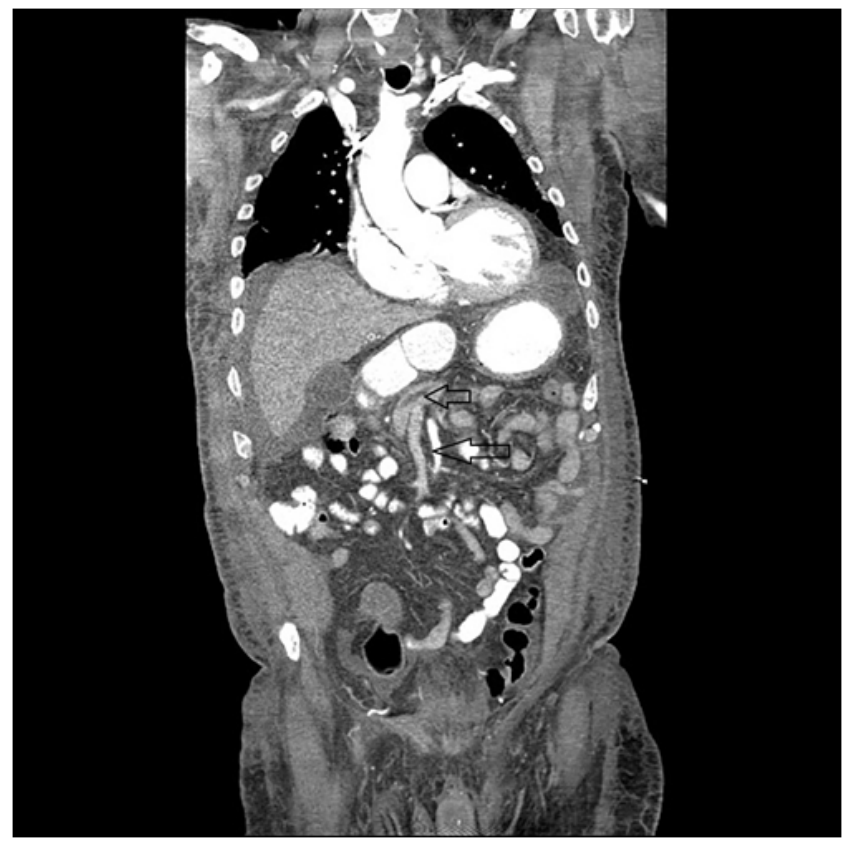

Figure 3 CT scan of the abdomen showing air/gas lucencies noted within the distal main and left portal veins.

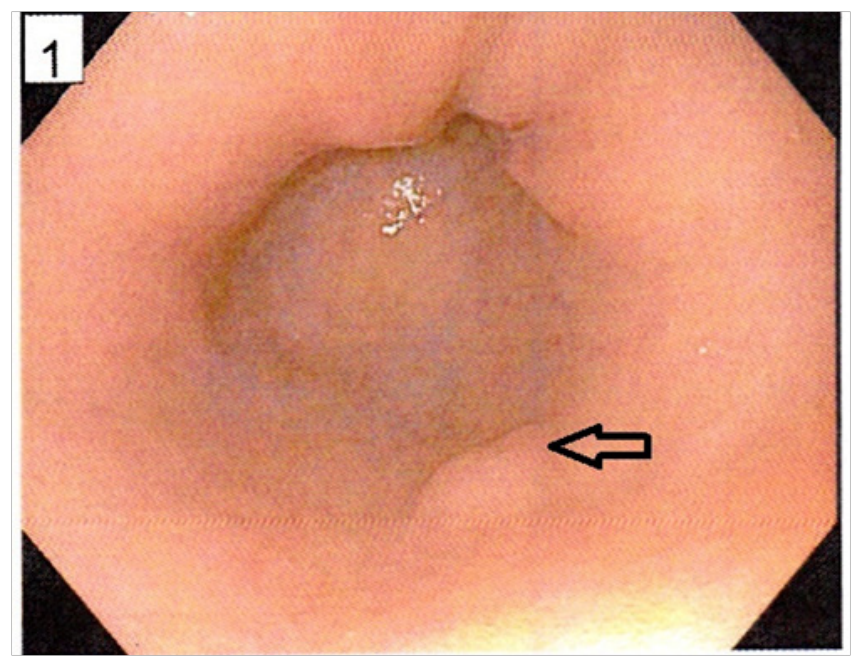

Figure 4 EGD showed mildly erythematous mucosa which appeared suggestive of mild gastritis in antrum of the stomach and a single smooth nodule measuring 5-6 mm in size in the antrum( white arrow).

\section{Discussion}

We present (Case 1) a case of elderly patient with gastric emphysema in setting of gastric ischemia. The interruption of blood supply to the stomach was likely due to transient strangulation of sliding hernia after a heavy meal. Despite the worrisome finding of imaging, our patient was managed with conservative treatment with bowel rest and intravenous fluid. We again present (Case 2) a case of elderly patient with emphysematous gastritis with air in the portal venous system in setting of septic shock most likely from right thigh abscess .In spite of the ominous signs, the decision was opted for conservative treatment.

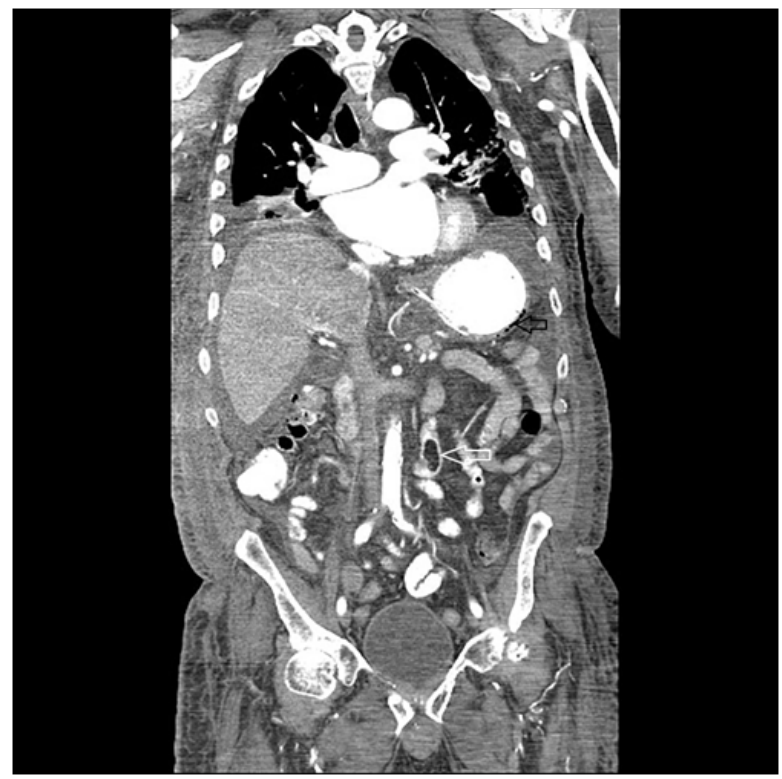

Figure $5 \mathrm{CT}$ scan of the abdomen showing (a) presence of gastric wall pneumatosis (black arrow) and (b) $2.6 \mathrm{~cm}$ small bowel lipoma (white arrow).

The collection of gas in the wall of stomach can be found in three circumstances: (1) interstitial gastric emphysema (GE), (2) cystic pneumatosis and (3) emphysematous gastritis (EG). ${ }^{1}$ Although less than 100 cases of both gastric emphysema and emphysematous gastritis have been found in last three decades, only 18 cases of GE is recorded in 15 years of period ${ }^{2}$ and 68 cases of EG has been reported in the literature: 32 cases before $20^{\text {th }}$ century and another 27 cases after $20^{\text {th }}$ century. ${ }^{3}$ The very first case of EG was recorded by Fraenkel ${ }^{4}$ in 1889 .

The presence of intramural gas in the stomach is recognized as gastric emphysema (GE). They usually are rare but alarming radiological finding. Gastric emphysema can be caused by mechanical forces such as nasogastric tube insertion, emesis, alveolar air leaks through mediastinum in chronic obstructive pulmonary disease, chemotherapy induced mucosal ischemia and bacterial infections such as pseudomonas. ${ }^{5}$ The finding of round gas bubble in the stomach on the imaging is pathognomonic features of gastric emphysema. Clinically, the patients are more stable compared to emphysematous gastritis.

Cystic emphysema is defined as the presence of several $1-2 \mathrm{~mm}$ gas filled cysts in gastric wall on radiological images. They usually present with mild gastrointestinal symptoms. In emphysematous gastritis, the characteristic radiological finding is presence of streaky and linear air in gastric intramural area. Patients present with severe abdominal pain associated with fever, leukocytosis and complications such as hematemesis and melena. They are more critically ill and toxic looking.

The mortality rate from EG was nearly $60 \%$.However, it has been decreased to nearly $33 \%$ after $20^{\text {th }}$ century. In Watson et al., ${ }^{3}$ EG before 
Year 2000 has managed with exploratory laparotomy while early endoscopic stratification with EGD and optimal medical treatment is preferred after Year 2000. The advancement in diagnostic techniques and changing trends after 2000 most likely improve survival rate in EG. The patient with EG may usually present in a week to as late as six weeks after triggering event. ${ }^{6}$ The common organisms associated with EG are poly-microbial, clostridium species and candida species. The triggering factors for EG includes corrosives, alcohol abuse, non-steroidal anti-inflammatory medications, recent surgery, sepsis, gastroenteritis. ${ }^{6,7}$

Elevated serum lactate is associated with bad prognosis in gastric pneumatosis. ${ }^{8}$ Gastric pneumatosis should be considered as a rare but possible differential diagnosis on presenting with abdominal pain with leukocytosis superimposed by emesis, CAT scan remains the best imaging for diagnosis in all cases. The presence of air in the mucosa and submucosa in any part of gastrointestinal tract is known as the pneumatosis. The causes that lead to gastrointestinal pneumatosis include ischemia, diverticulitis, duodenal ulcers, duodenal stenosis, gastric outlet obstruction, AIDS, steroids, vasculitis, connective tissue disorders, inflammatory bowel disease, or rarely tumors .However, ischemia is the most common cause. ${ }^{9-11}$ Pneumatosis itself does not represent underlying severe pathologic conditions. However, it is associated with succumb prognosis and high mortality rate. ${ }^{12}$

Surgical intervention can be considered depending on presentation, hemodynamic status, age, comorbidities, extent of bowel involvement, complications such as perforation, peritonitis, and necrotic bowel. Despite the surgical intervention, the outcome is usually poor and unsatisfactory in setting of comorbidities.

\section{Conclusion}

In our case series, (Case 1) gastric emphysema is caused by gastric ischemia from interruption of blood supply to the stomach due to transient strangulation of the sliding hernia after heavy meal. Case 2 emphysematous gastritis with air in the portal venous system caused by bowel ischemia from hypo-perfusion in setting of septic shock most likely from right thigh abscess and vasopressors. In spite of the worrisome radiological findings, conservative management was appreciated for both cases.

\section{Author Contribution}

Dr. Kyawzaw Lin contributed equally in gathering information, writing introduction, case report and discussion. Dr. Aung Naing Lin, Dr. Andrew Ofosu, Dr. Emanuel Ofori, Dr. Sandar Linn and Dr. Madhavi Reddy involved in editing the abstract. Dr. Moshe Fuksbrumer provided the radiological image of the report.

\section{Acknowledgements}

None.

\section{Funding}

None.

\section{References}

1. D'Cruz R, Emil S. Gastroduodenal emphysema. J Pediatr Surg. 2008;43(11):2121-2123.

2. Johnson PT, Horton KM, Edil BH, et al. Gastric Pneumatosis:the role of $\mathrm{CT}$ in diagnosis and patient management. Emerg Radiol. 2011;18(1):65-73

3. Watson A, Bul V, Staudacher J, et al. The predictors of mortality and secular changes in management strategies in emphysematous gastritis. Clin Res Hepatol Gastroenterol. 2017;41(1):e1-e7.

4. Fraenkel E. Ueber einen Fall von Gastritis acuta emphysematosa wahrscheinlich mykotischen Ursprungs. Archiv für pathologische Anatomie und Physiologie und für klinische Medicin. 1889;118(3):526-535.

5. Van Mook WN, van der Geet S, Goessens ML, et al. Gas within the wall of the stomach due to emphysematous gastritis : case report and review. Eur J Gastroenterol hepatol. 2002;14(10):1155-1160.

6. Moosvi AR, Saravolatz LD, Wong DH, et al. Emphysematous gastritis: case report and review. Rev Infect Dis. 1990;12(5):848-855.

7. Badrinath K, Jairam N, Ravi HR, et al. Gastric emphysema following drug-induced gastritis. British journal of surgery. 1992;79(4):320.

8. Hawn MT, Canon CL, Lockhart ME, et al. Serum lactic acid determines outcomes of CT diagnosis of pneumatosis of the gastrointestinal tract. AM Surg. 2004;70(1):19-23.

9. Lunca S, Rikkers A, Stãnescu A. Acute massive gastric dilatation: severe ischemia and gastric necrosis without perforation. Rom J Gastroenterol .2005;14(3):279-283.

10. Kataria R, Bhatnagar V, Wadhwa $\mathrm{S}$, et al. Gastric pneumatosis associated with preduodenal portal vein, duodenal atresia, and asplenia. Pediatr Surg Int. 1998;14(1-2):100-101.

11. Kawano S, Tanaka H, Daimon Y, et al. Gastric pneumatosis associated with duodenal stenosis and malrotation. Pediatric radiology. 2001;31(9):656-658.

12. Muhammad MN, Sadough M, King R, et al. Pneumatosis of the esophagus and intestines with portal venous air: a rare presentation. $J$ Community Hosp Intern Med Perspect. 2017;7(4):255-257.

\section{Conflicts of Interest}

None. 